\title{
Características iniciais da comunicação verbal de pré- escolares com Alterações Específicas do Desenvolvimento da Linguagem em fala espontânea
}

\author{
Primary characteristics of the verbal communication \\ of preschoolers with Specific Language Impairment in \\ spontaneous speech
}

Debora Maria Befi-Lopes ${ }^{1}$, Silmara Rondon²

\begin{abstract}
RESUMO
Objetivo: Verificar desempenho fonológico de pré-escolares com Alterações Específicas do Desenvolvimento da Linguagem (AEDL) em fala espontânea. Métodos: Foram sujeitos 27 crianças com AEDL, entre três anos e cinco anos e 11 meses, em tratamento fonoaudiológico. Foram selecionados aqueles que realizaram ao menos $50 \%$ da avaliação da fonologia a partir de provas de nomeação e imitação de palavras, ou que apresentaram inteligibilidade de fala passível de análise. Foram coletadas amostras de fala na prova de pragmática e no discurso eliciado por figuras. Foram realizadas análises a partir da utilização de processos fonológicos do desenvolvimento de linguagem (PD) e idiossincráticos (PI). Resultados: A estatística descritiva (médias de PD e PI) indicou grande variabilidade intra-grupos. Não houve variação em número de processos conforme a idade ( $P D$ : p=0,38; PI: p=0,72), porém houve predominância de PD em todas as idades, nas duas provas aplicadas $(Z=-6,327 ; p<0,001)$. A ocorrência de PD e PI foi maior na prova de pragmática $(\mathrm{p}<0,001)$, situação em que o número de palavras produzidas também foi maior ( $\mathrm{T}$-valor=8,93; $\mathrm{p}=0,000)$. Conclusão: $\mathrm{A}$ grande variabilidade intra-grupo confirma a heterogeneidade dos quadros de AEDL. Pode-se atribuir a ininteligibilidade, que dificulta a avaliação da linguagem expressiva desses sujeitos, à ocorrência de PD e PI concomitantemente. Ademais, a interação desenvolvida durante a prova de pragmática mostrou-se mais efetiva para a obtenção de amostra de fala espontânea para a análise da fonologia, além de confirmar a existência de grandes dificuldades relacionadas à elaboração de idéias e sua expressão em sujeitos com AEDL.
\end{abstract}

Descritores: Desenvolvimento da linguagem; Fala; Avaliação; Criança; Pré-escolar

\section{INTRODUÇÃO}

As Alterações Específicas do Desenvolvimento da Linguagem (AEDL) caracterizam-se como quadros de alterações na aquisição e no desenvolvimento das habilidades de linguagem em que não ocorrem outros déficits, os quais

Trabalho realizado no Laboratório de Investigação Fonoaudiológica em Desenvolvimento da Linguagem e suas Alterações do Curso de Fonoaudiologia do Departamento de Fisioterapia, Fonoaudiologia e Terapia Ocupacional da Faculdade de Medicina da Universidade de São Paulo - USP - São Paulo (SP), Brasil.

(1) Livre-docente, Professora Associada do Curso de Fonoaudiologia da Faculdade de Medicina da Universidade de São Paulo - USP - São Paulo (SP), Brasil.

(2) Pós-graduanda (Mestrado) em Ciências da Reabilitação do Departamento de Fisioterapia, Fonoaudiologia e Terapia Ocupacional da Faculdade de Medicina da Universidade de São Paulo - USP - São Paulo (SP), Brasil. Endereço para correspondência: Debora Maria Befi Lopes. R. Cipotânea, 51, Cidade Universitária, São Paulo (SP), Brasil, CEP: 05360-160. E-mail: dmblopes@usp.br

Recebido em: 00/00/2009; Aceito em: 00/00/2009 poderiam contribuir para a instalação deste distúrbio ${ }^{(1,2)}$. Esses indivíduos progridem adequadamente em outras áreas que não a linguagem ${ }^{(3)}$.

O diagnóstico do referido distúrbio é dado com base em fatores de exclusão, tendo como fator de inclusão o baixo desempenho em testes padronizados de linguagem ${ }^{(4)}$. Esse diagnóstico caracteriza uma classe muito heterogênea de indivíduos que possuem diferentes dificuldades em linguagem, seja na sua aquisição ou desenvolvimento, as quais podem ocorrer por diversas razões, ainda pouco estudadas ${ }^{(5-7)}$.

Diferentemente dos quadros de Transtorno Fonológico, encontram-se dados na literatura afirmando que, nos quadros de AEDL, os vários subsistemas da linguagem estão comprometidos (semântica, morfossintaxe, pragmática); entretanto, por ocasião do diagnóstico em linguagem, um dos componentes que pode estar mais afetado é a fonologia ${ }^{(2,8-11)}$.

Sujeitos com AEDL preferem produzir palavras que contenham fonemas que já fazem parte de seu inventário fonético. Assim, selecionam tipos lexicais nos quais seus sistemas 
de produção se baseiam, evitando produzir palavras menos conhecidas ${ }^{(12-14)}$.

Dessa maneira entende-se que uma das melhores amostras de fala para análise da performance linguística nas AEDL é a amostra de fala espontânea, indireta e a partir de uso funcional da linguagem em interação lúdica. Esse tipo de amostra tem a vantagem de retratar o comportamento natural do indivíduo, o qual trabalha com situações inesperadas e executa suas funções de linguagem de acordo com o contexto proposto no momento da interação comunicativa ${ }^{(15)}$.

Ademais, foi constatado que para a avaliação da linguagem nas AEDL é importante que sejam consideradas as primeiras ocasiões em que os indivíduos que têm o distúrbio produzem discurso, ou seja, as primeiras ocasiões em que estes começam a combinar palavras e realizar pequenas sentenças. Isso porque é nessa fase que esses indivíduos começam a apresentar de fato dificuldades relacionadas, principalmente, à fonologia (pouca precisão articulatória na produção dos sons, dificuldades de segmentação, entre outros), como também em sintaxe e pragmática (o uso funcional da linguagem fica geralmente comprometido $)^{(16-20)}$.

Nos estágios iniciais da aquisição da linguagem por essas crianças, as alterações fonológicas comprometem a avaliação dos demais subsistemas, uma vez que a ininteligibilidade de fala é comum, prejudicando a avaliação da linguagem de forma geral $^{(13,14)}$.

Assim, os objetivos do presente trabalho foram verificar o desempenho fonológico de pré-escolares com diagnóstico de Alteração Específica do Desenvolvimento da Linguagem Retardos na Aquisição da Linguagem e Distúrbios Específicos de Linguagem - no que se refere aos padrões de produção em fala espontânea, em duas situações diferentes de coleta, bem como comparar a performance dessas crianças em cada uma das situações, com o desempenho descrito para crianças de mesma faixa etária em desenvolvimento normal, da cidade de São Paulo(21).

\section{MÉTODOS}

Foram sujeitos desta pesquisa 27 crianças que procuraram atendimento fonoaudiológico no Laboratório de Investigação Fonoaudiológica em Desenvolvimento da Linguagem e suas Alterações da Faculdade de Medicina da Universidade de São Paulo (USP), ou que estiveram em processo terapêutico semanal durante a vigência da coleta de dados, os quais foram incluídos obedecendo aos seguintes critérios:

- autorização dos pais e/ou responsáveis para participação na pesquisa;

- respeitar os critérios de exclusão para diagnóstico de Alterações Específicas do Desenvolvimento da Linguagem, a saber: perdas auditivas, lesões cerebrais focais ou disfunções neurológicas adquiridas, comprometimento motor do desenvolvimento de fala, distúrbios abrangentes do desenvolvimento ou síndromes ${ }^{(1,2)}$, tendo como fator de inclusão apenas o baixo desempenho em testes padronizados de linguagem ${ }^{(4)}$;

- faixa etária entre três anos e cinco anos e 11 meses, de ambos os gêneros
- possuir, no mínimo, oralidade suficiente para a realização das provas de discurso e emitir produções orais analisáveis, ou seja, aquelas que fossem passíveis de transcrição compreensível na prova de pragmática ${ }^{(22)}$. Sujeitos cujas produções orais não permitiam transcrição fonológica, não puderam compor o grupo aqui estudado, uma vez que nosso objetivo foi descrever as características fonológicas da primeira amostra analisável de fala.

Para a realização do presente estudo foram necessários:

- um gravador digital com cabo USB para gravação fidedigna das emissões das crianças nas provas de imitação e nomeação;

- um microfone de lapela acoplável ao gravador;

- brinquedos para realização de interação lúdica com as crianças a fim de coletar fala espontânea (prova de pragmática);

- figura para coleta do discurso (aqui utilizamos uma figura de festa de aniversário, ou a figura para eliciação de discurso do ABFW - Teste de Linguagem Infantil ${ }^{(23)}$ e utilizamos na transcrição a amostra de fala que apresentou o maior número de palavras inteligíveis e passíveis de análise);

- uma filmadora digital, pois na maioria dos casos, só é possível a transcrição da fala espontânea a partir da observação da situação, ou seja, do contexto comunicativo, uma vez que a ininteligibilidade de fala pode impedir a determinação de se aquela emissão refere-se, ou não, à temática da interação;

- 100 DVD-RW para a gravação da coleta de fala espontânea; - um notebook Pentium 4.

Após a seleção dos sujeitos, como já descrito na casuística deste mesmo item de nosso projeto, foi realizada a coleta de dados. Todas as crianças que se caracterizaram como sujeitos da pesquisa foram primeiramente avaliadas por meio de provas específicas de avaliação da fonologia - nomeação de figuras e imitação de palavras ${ }^{(21)}$, em situação dirigida, dentro da sala de terapia que estão acostumadas a frequentar. Para evitar possível cansaço na realização das provas, estas foram aplicadas em dias separados (com diferença de sete a quinze dias entre uma avaliação e outra, sendo que $50 \%$ das crianças fizeram primeiro a prova de imitação e $50 \%$ primeiro a prova de nomeação).

Após a aplicação destas provas, todos os sujeitos que conseguiram realizar pelo menos $50 \%$ do total de cada uma das provas, foram gravados (em outro dia), em situação de interação lúdica com a pesquisadora (prova de pragmática ${ }^{(22)}$ ) e coleta da fala espontânea a partir de figura (discurso), a fim de se realizar o levantamento para posterior análise fonológica, seguindo os mesmos parâmetros, a saber:

- utilização de processos fonológicos comuns ao desenvolvimento normal da linguagem (parâmetros para o Português do Brasil $^{(21)}$ ), em comparação a seus pares de mesma faixa etária. Esses processos fonológicos são divididos em: redução de sílaba, harmonia consonantal, plosivação de fricativa, frontalização de velar, posteriorização para velar, posteriorização para palatal, frontalização de palatal, simplificação de líquida, simplificação do encontro consonantal, simplificação da consoante final, sonorização de plosivas, sonorização de fricativas; - utilização de processos fonológicos idiossincráticos ${ }^{(13)}$ 
Tabela 1. Estatística descritiva para processos fonológicos do desenvolvimento (PD) e processos fonológicos de não-desenvolvimento (PI), por idade

\begin{tabular}{cccccccc}
\hline & Idade & N & Média & Mediana & DP & Mínimo & Máximo \\
\hline PD & 3 & 6 & 25,08 & 18,50 & 18,60 & 3,00 & 53,00 \\
& 4 & 12 & 27,50 & 27,50 & 24,72 & 0 & 88,00 \\
& 5 & 9 & 42,83 & 42,83 & 40,39 & 4,00 & 158,00 \\
\hline PI & 3 & 6 & 7,50 & 5,50 & 6,42 & 0 & 22,00 \\
& 4 & 12 & 9,88 & 5,50 & 12,12 & 0 & 47,00 \\
& 5 & 9 & 10,83 & 9,50 & 10,14 & 1,00 & 39,00 \\
\hline
\end{tabular}

Legenda: $\mathrm{PD}$ = processos fonológicos do desenvolvimento; $\mathrm{PI}=$ processos fonológicos de não-desenvolvimento; $\mathrm{DP}$ = desvio-padrão; $\mathrm{N}$ = número de sujeitos

(não descritos no desenvolvimento normal da linguagem). Esses processos fonológicos são divididos em: eliminação da consoante inicial, omissão de semivogal, ensurdecimento de plosivas, troca de vogais, ensurdecimento de fricativas, frontalização de alveolar, posteriorização para alveolar, omissão de vogal, adição de sílaba, nasalização e denasalização. Além desses, foram também analisados os processos fonológicos idiossincráticos de sonorização de plosivas e sonorização de fricativas ${ }^{(21)}$.

Após a coleta e transcrição dos dados, estes foram tabulados respeitando-se a análise proposta no item procedimento do presente estudo e passaram por tratamento estatístico cabível para respostas aos nossos objetivos.

O presente estudo não apresentou riscos para a população que foi avaliada, tendo sido aprovado pela Comissão de Ética em Pesquisa da instituição tanto no que se refere ao seu teor bem como aos aspectos constantes do Termo de Consentimento Livre e Esclarecido assinado pelos responsáveis pelas crianças que foram estudadas (protocolo de autorização número 535/06).

\section{RESULTADOS}

Para a análise estatística dos dados referentes a este estudo foram utilizados os seguintes testes não-paramétricos: Quiquadrado, Wilcoxon e Kruskall-Wallis (teste de hipótese). Foram utilizadas técnicas não-paramétricas para análise estatística dos dados, pois estas são mais indicadas nos casos em que os dados coletados não oferecem as condições básicas para a realização de testes paramétricos, dada a heterogeneidade das variâncias apresentada. Além disso, foi utilizado o teste $\mathrm{T}$ para análise inferencial dos dados. $\mathrm{O}$ nível de significância adotado foi de $5 \%$.

A Tabela 1 mostra a estatística descritiva geral da produtividade de PD e PI para cada faixa etária. É possível observar, pelo desvio-padrão e valores mínimos e máximos, que a variabilidade intra-grupos de idade foi muito alta, o que pode refletir a heterogeneidade do quadro clínico da população estudada.

$\mathrm{O}$ teste não-paramétrico mostrou que o número de processos fonológicos do desenvolvimento (PD) e de não-desenvolvimento (PI), independente da prova, não variou com a idade, dado que o p-valor obtido não foi significativo ( $\mathrm{PD}$ : $\mathrm{H}=1,92$; $\mathrm{p}=0,383$; PI: $\mathrm{H}=0,66 ; \mathrm{p}=0,721$ ).

A Tabela 2 apresenta as comparações entre a ocorrência de PD e PI de acordo com as faixas etárias, levando-se em conta as provas realizadas (duas situações de fala espontânea consideradas - pragmática e discurso), não indicando diferença entre elas.
Tabela 2. Comparação entre as faixas etárias, levando-se em conta a prova e os processos

\begin{tabular}{llcc}
\hline Processo & Prova & $\mathrm{H}$ & Valor de $\mathrm{p}$ \\
\hline $\mathrm{PD}$ & Pragmática & 4,19 & 0,123 \\
& Discurso & 1,05 & 0,591 \\
\hline $\mathrm{PI}$ & Pragmática & 1,24 & 0,538 \\
& Discurso & 0,13 & 0,939 \\
\hline
\end{tabular}

Teste Wilcoxon

Legenda: $\mathrm{PD}=$ processos fonológicos do desenvolvimento; $\mathrm{PI}=$ processos fonológicos de não-desenvolvimento

A comparação entre o número de PD e PI, independente da idade e das provas, indicou predominância para ocorrência de PD nas duas situações de fala espontânea consideradas, para todas as faixas etárias $(\mathrm{Z}=-6,327 ; \mathrm{p}<0,001)$.

Na Tabela 3, pode-se observar a análise da ocorrência de processos fonológicos por prova e por tipologia (PD X PI). $\mathrm{A}$ análise indicou que as provas se diferenciaram significativamente entre si quanto ao número de PD e de PI, sendo maior na prova de pragmática. Além disso, o número de PD foi estatisticamente maior que a de PI em ambas as provas.

Tabela 3. Comparação entre os processos por prova e por tipologia

\begin{tabular}{lcccc}
\hline & PDd x PDp & PId x PIp & PDp x PIp & PDd x PId \\
\hline$Z$ & $-4,458$ & $-4,543$ & $-4,542$ & $-4,441$ \\
Valor de $p$ & $<0,001^{*}$ & $<0,001^{*}$ & $<0,001^{*}$ & $<0,001^{*}$ \\
\hline
\end{tabular}

Teste Qui-quadrado com diferença entre duas proporções

*Valores significativos $(p \leq 0,05)$

Legenda: $P D d=$ processo do desenvolvimento na prova de discurso; $P D p=$ processo do desenvolvimento na prova de pragmática; $\mathrm{PId}=$ processo idiossincrático na prova de discurso; $\mathrm{Plp}$ = processo idiossincrático na prova de pragmática

Ademais, foi possível observar a diferença, comprovada pelo teste $\mathrm{T}$, entre o número de palavras produzidas durante a prova de pragmática, que foi maior do que o número de palavras produzidas na prova de discurso (T-valor $=8,93 ; \mathrm{p}=0,000$ ). Esse dado indica que nesta pesquisa a prova de pragmática foi mais sensível para eliciar a fala espontânea das crianças, além de permitir análise mais precisa da ocorrência de PD e PI, quando comparada com a prova de discurso.

No que se refere à frequência de crianças que apresenta cada um dos PD em ambas as provas, de acordo com o teste Qui-quadrado, na prova da pragmática as crianças apresentaram mais harmonia consonantal $(\mathrm{HC})(\mathrm{p}=0,027)$, frontalização de velar $(\mathrm{FV})(\mathrm{p}=0,002)$, frontalização de palatal (FP) $(\mathrm{p}=0,001)$, simplificação de líquida $(\mathrm{SL})(\mathrm{p}=0,038)$, simplifica- 
ção do encontro consonantal (SEC) ( $<<0,001)$, simplificação da consoante final ( $\mathrm{SCF})(\mathrm{p}<0,001)$, sonorização de plosiva (SP) $(\mathrm{p}<0,001)$, sonorização de fricativa $(\mathrm{SF})(\mathrm{p}<0,001)$ e ensurdecimento de fricativa $(\mathrm{EF})(\mathrm{p}=0,029)$. Além disso, os valores de $\mathrm{p}$ encontrados para redução de sílaba (RS), plosivação de fricativa $(\mathrm{PF})$ e ensurdecimento de plosiva (EP) podem indicar uma tendência à diferença, que deveria ser confirmada, ou não, com o aumento da amostra.

Além disso, por meio da análise por idade, observou-se que, para as crianças de cinco anos, as frequências de RS e HC foram maiores $(p=0,020 ; p=0,011)$. Esse fator não parece ter sido influenciado pelo tempo em que os sujeitos se encontram em terapia, como se pode observar na Tabela 4.

Tabela 4. Comparação entre ocorrência de processos fonológicos do desenvolvimento de acordo com a situação

\begin{tabular}{lcccc}
\hline Situação & N & Mediana & H & Valor de p \\
\hline Diagnóstico & 10 & 18,50 & & \\
um ano de terapia & 13 & 31,00 & & \\
dois anos de terapia & 3 & 14,00 & 2,13 & 0,547 \\
três anos de terapia & 1 & 19,50 & & \\
\hline
\end{tabular}

Teste Kruskal-Wallis

Legenda: $\mathrm{N}$ = número de sujeitoS

No que se refere ao número e à frequência de crianças que apresentam cada um dos PI em ambas as provas, de acordo com o teste Qui-quadrado, na prova de pragmática as crianças apresentaram mais omissão de semivogal (OS) $(\mathrm{p}<0,001)$, troca de vogal $(\mathrm{TV})(\mathrm{p}=0,005)$, eliminação da consoante inicial (ECI) $(\mathrm{p}<0,001)$ e denasalização (D) $(\mathrm{p}=0,005)$. O número de processos fonológicos não passíveis de análise (NA) (aqueles que não puderam ser classificados segundo o proposto por Palmieri $\left.{ }^{(13)}\right)$ foi maior na prova de pragmática $(\mathrm{p}<0,001)$.

Ademais, pela análise por idade, foi possível observar que, para as crianças de cinco anos, a frequência de nasalização $(\mathrm{N})$ foi maior $(\mathrm{p}=0,021)$. Esse fator não parece ser influenciado pelo tempo de terapia em que se encontram os sujeitos, como se pode observar na Tabela 5.

Tabela 5. Comparação entre ocorrência de processos fonológicos de não-desenvolvimento de acordo com a situação

\begin{tabular}{lcccc}
\hline Situação & N & Mediana & H & Valor de p \\
\hline Diagnóstico & 10 & 7,00 & & \\
um ano de terapia & 13 & 6,00 & & \\
dois anos de terapia & 3 & 2,50 & 1,93 & 0,587 \\
três anos de terapia & 1 & 10,00 & & \\
\hline
\end{tabular}

Teste Kruskal-Wallis

Legenda: $\mathrm{N}$ = número de sujeitos

\section{DISCUSSÃO}

A partir dos dados encontrados no presente trabalho, foi possível destacar algumas considerações acerca do desenvolvimento fonológico de crianças com Alterações Específicas do Desenvolvimento da Linguagem (AEDL).

As análises dos resultados em geral possibilitaram a ob- servação de um alto índice de variabilidade, o que corrobora os dados da literatura e com a experiência clínica, acerca da heterogeneidade dos quadros de $\mathrm{AEDL}^{(5-7)}$, como pode ser observado na Tabela 1.

Neste estudo as crianças com AEDL apresentaram processos fonológicos do desenvolvimento assim como é esperado para crianças em desenvolvimento típico. No entanto, foi possível observar que, mesmo com o aumento da idade, houve a manutenção de processos fonológicos que já deveriam ter sido superados ${ }^{(21,24,25)}$.

Os processos fonológicos redução de sílaba (RS) e harmonia consonantal (HC) foram apresentados pelas crianças nas faixas etárias estudadas, permanecendo até os cinco anos, nas duas situações de fala espontânea coletadas. Esses processos fonológicos devem ser eliminados até por volta dos dois anos e seis meses (segundo parâmetros para o Português do Brasil $^{(21)}$ ) e todos os sujeitos da pesquisa encontravam-se na faixa etária entre três anos e cinco anos e 11 meses, ou seja, todos já deveriam tê-los superado. Confirma-se então o atraso no desenvolvimento fonológico dessas crianças, discutido na literatura $^{(2,8-11)}$.

Ademais, foram também significativamente apresentados os processos fonológicos frontalização de velar (FV), simplificação de líquida (SL) (os quais devem ser eliminados até os três anos e seis meses), sonorização de plosiva (SP), sonorização de fricativa (SF) e ensurdecimento de fricativa (EF), para todas as faixas etárias estudadas. Eram esperados somente os processos simplificação do encontro consonantal (SEC) e simplificação da consoante final (SCF), visto que devem ser eliminados por volta dos sete anos de idade, no desenvolvimento típico, mas que foram apresentados em todas as idades nesta pesquisa, em número significativo(21).

Além da manutenção dos PD, foi também observada a ocorrência de PI para todas as faixas etárias estudadas, nas duas situações de fala espontânea propostas. Esse dado evidencia a imaturidade fonológica dessas crianças, que apresentam tanto PD como PI durante o desenvolvimento da linguagem, em que um dos subsistemas mais afetados é a fonologia ${ }^{(4,13)}$.

Dentre os PI apresentados, são encontrados em número significativos os processos de omissão de sílaba (OS), troca de vogal (TV), eliminação da consoante inicial (ECI) e denasalização (D). Encontra-se na literatura que, quando há ocorrência dos processos OS e ECI, estes costumam ser eliminados com o aumento da idade, ocorrendo em maior número em crianças em fases iniciais do desenvolvimento da linguagem ${ }^{(26-28)}$.

Ademais, foi estatisticamente significante o número de processos fonológicos apresentados nas amostras das duas diferentes situações de fala espontânea que não foram passíveis de análise, segundo as classificações aqui utilizadas. Esse último dado mostra que foi grande a ininteligibilidade de fala apresentada nos casos de AEDL aqui estudados, em que a fonologia estava comprometida, pois, além de apresentarem PD e PI, essas crianças apresentaram processos fonológicos não passíveis de análise. Esse fato dificulta as análises de fala para identificação de outras alterações da linguagem, além daquelas referentes à fonologia ${ }^{(13,14)}$.

Em estudo comparativo entre o desempenho lexical e 
fonológico em crianças com AEDL foram encontrados dados referentes à ocorrência de processos fonológicos não passíveis de análise ${ }^{(13)}$, os quais podem indicar uma interferência das questões relativas ao desenvolvimento lexical no desempenho destes sujeitos durante avaliação fonológica.

No que se refere ao número de ocorrências de PD e PI, a ocorrência de PD foi maior do que a de PI, independentemente da faixa etária estudada, do tempo de terapia ou da prova analisada, indicando predominância dos PD na fala espontânea.

Ademais, os valores mínimos e máximos para a ocorrência de PD e PI são muito variáveis dentro do grupo estudado, independentemente de sua faixa etária e da situação de fala espontânea selecionada para análise (pragmática ou discurso). Esses dados evidenciam a heterogeneidade encontrada no perfil linguístico geral dessas crianças, além de demonstrar as diferenças encontradas no perfil fonológico nas AEDL, comparado àquele encontrado no desenvolvimento típico ${ }^{(13,14)}$.

A grande variação individual observada no desempenho fonológico dos sujeitos da pesquisa, demonstrada pela análise quantitativa, como também a partir dos dados observados qualitativamente, indica que essas crianças apresentam desenvolvimento fonológico atípico, muitas vezes encontrando-se em estágios primitivos de desenvolvimento do sistema fonológico e da linguagem em geral, como tem sido discutido na literatura em relação às $\operatorname{AEDL}^{(6,9,13,14)}$.

Neste estudo, a amostra de fala espontânea coletada por meio da prova de pragmática ${ }^{(22)}$ mostrou-se mais eficiente para a identificação da ocorrência tanto de PD como de PI, sendo considerada mais sensível para a caracterização do perfil fonológico de crianças com AEDL. Outro dado importante é que as amostras de fala coletadas por meio do discurso continham menor número de palavras, diminuindo assim a possibilidade de ocorrência de processos fonológicos. Isso remete à constatação de que crianças com AEDL costumam evitar a produção de palavras que contenham fonemas que ainda não fazem parte de seu inventário fonético, apresentando também dificuldades relacionadas à elaboração de narrativas ${ }^{(29,30)}$.

\section{CONCLUSÃO}

Os dados obtidos nesta pesquisa mostraram que foi grande o déficit fonológico de pré-escolares com AEDL, quando comparados aos seus pares cronológicos em desenvolvimento típico, o que prejudica significativamente seu desempenho em linguagem expressiva. Foi possível também identificar e confirmar a grande heterogeneidade desta população, sobretudo no que se refere ao seu perfil fonológico.

Os pré-escolares aqui estudados, como já indicado na literatura, no momento em que foram avaliados, apresentaram predominantemente processos fonológicos do desenvolvimento (PD), assim como apresentam pré-escolares em desenvolvimento típico. No entanto, o que foi observado é que os sujeitos com AEDL tenderam a manter os referidos processos em etapas tardias do desenvolvimento de linguagem, o que não acontece durante o desenvolvimento típico.

Além disso, foi grande a ocorrência de processos fonológicos idiossincráticos (PI) nas duas situações de fala espontânea propostas, juntamente com os PD e processos fonológicos não passíveis de análise. Esses dados confirmam o que se apresenta na literatura, no que se refere ao grau de comprometimento da inteligibilidade de fala dessas crianças em contextos de interação verbal não dirigida.

Ademais, foi possível verificar que o número de palavras produzidas e processos fonológicos apresentados foi maior nas amostras de fala espontânea obtidas a partir da prova de pragmática, quando comparadas àquelas obtidas a partir do discurso eliciado por figura. Esse dado evidencia que aquele tipo de interação é mais efetivo para a obtenção de amostra de fala espontânea para a análise da fonologia, além de confirmar a existência de grandes dificuldades relacionadas à elaboração de idéias e sua expressão em narrativas em sujeitos com AEDL.

\section{AGRADECIMENTOS}

Agradecemos à Fundação de Amparo à Pesquisa do Estado de São Paulo (FAPESP), pelo apoio concedido para realização dessa pesquisa, sob processo de número 06/61458-0.

\begin{abstract}
Purpose: To verify the phonological performance of preschoolers with Specific Language Impairment (SLI) in spontaneous speech. Methods: The subjects were 27 children with SLI with ages between three years and five years and 11 months, who attended Speech-Language Pathology therapy. The subjects who carried out at least $50 \%$ of the phonological assessment or who had speech intelligibility that allowed analysis were selected. Speech samples were obtained from a pragmatics evaluation and from elicited discourse. Analyses considered the use of developmental (DP) and idiossyncratic phonological processes (IP) in spontaneous speech. Results: The descriptive statistics (mean DP and IP) showed large within-group variability. There was no variation in the number of processes according to age (DP: $\mathrm{p}=0.38$; IP: $\mathrm{p}=0.72)$, but there was a prevalence of $\mathrm{DP}$ in all ages, in both tests $(\mathrm{Z}=-6.327 ; \mathrm{p}<0.001)$. The occurrence of DP and IP was higher in the pragmatics evaluation $(\mathrm{p}<0.001)$, situation in which the number of words produced was also greater ( $\mathrm{T}$-value $=8.93 ; \mathrm{p}=0.000$ ). Conclusion: The great within-group variability confirms the heterogeneity of SLI. The speech unintelligibility, which hampers the assessment of the expressive language of these subjects, can be attributed to the cooccurrence of DP and IP. Moreover, the interaction during the pragmatics evaluation was more effective for obtaining a sample of spontaneous speech for phonological analysis, and confirms the existence of major difficulties related to the development of ideas and their expression in subjects with SLI.
\end{abstract}

Keywords: Language development; Speech; Evaluation; Child; Child preschool 


\section{REFERÊNCIAS}

1. Bortollini U, Leonard LB. Phonology and children with specific language impairment: status of structural constraints in two languages. J Commun Disord. 2000;33(2):131-49; quiz 149-50.

2. Befi-Lopes DM. Avaliação, diagnóstico e aspectos terapêuticos nos distúrbios específicos de linguagem. In: Ferreira LP, Befi-Lopes DM, Limongi SCO. Tratado de fonoaudiologia. São Paulo: Roca; 2004. p. 987-1000

3. Baddeley A, Gathercole S, Papagno C. The phonological loop as a language learning device. Psychol Rev. 1998;105(1):158-73.

4. Befi-Lopes DM, Palmieri TM. Análise dos processos fonológicos utilizados por crianças com alteração no desenvolvimento da linguagem. J Bras Fonoaudiol. 2000;1(4):48-58.

5. Watkins RV. Specific language impairments in children: an introduction. In: Watkins RV, Rice ML, editors. Specific language impairments in children. Baltimore: P. H. Brookes Pub.; c1994. p. 1-34.

6. McDonald JL. Language acquisition: the Acquisition of linguistic structure in normal and special populations. Annu Rev Psychol. 1997;48:215-41.

7. Tager-Flusberg H, Cooper J. Present and future possibilities for defining a phenotype for specific language impairment. J Speech Lang Hear Res. 1999;42(5):1275-8.

8. Wagner CR, Nettelbladt U, Sahlén B, Nilholm C. Conversation versus narration in pre-school children with language impairment. Int J Lang Commun Disord. 2000;35(1):83-93.

9. Orsolini M, Sechi E, Maronato C, Bonvino E, Corcelli A. Nature of phonological delay in children with specific language impairment. Int $\mathrm{J}$ Lang Commun Disord. 2001;36(1):63-90.

10. Joanisse MF, Seidenberg MS. Phonology and syntax in specific language impairment: evidence from a connectionist model. Brain Lang. 2003;86(1):40-56.

11. Newbury DF, Bishop DV, Monaco AP. Genetic influences in language impairment and phonological short-term memory. Trends Cogn Sci. 2005;9(11): 528-34.

12. Leonard LB, Bortolini U, Caselli MC, McGregor KK, Sabbadini L. Morphological deficits in children with specific language impairment: the status of features in the underlying grammar. Lang Acqu. 1992;2(2):151-79.

13. Palmieri TM. Comparação entre o desempenho fonológico e lexical de crianças com alteração do desenvolvimento da linguagem [dissertação]. São Paulo: Faculdade de Filosofia Letras e Ciências Humanas da Universidade de São Paulo; 2002.

14. Befi-Lopes DM, Gândara JP, Araújo K. Aquisição do sistema fonológico em crianças com alterações no desenvolvimento da linguagem. Pró-Fono. 2003;15(1):19-30.

15. Hewitt LE, Hammer CS, Yont KM, Tomblin JB. Language sampling for kindergarten children with and without SLI: mean length utterance, IPSYN, and NDW. J Commun Disord. 2005;38(3):197-213.
16. Bishop DV, Chan J, Adams C, Hartley J, Weir F. Conversational responsiveness in specific language impairment: evidence of disproportionate pragmatic difficulties in subset of children. Dev Psychopathol. 2000;12(2):177-99. Erratum in: Dev Psychopathol. 2008;20(1):399.

17. Befi-Lopes DM, Rodrigues A, Rocha LC. Habilidades linguísticopragmáticas em crianças normais e com alterações de desenvolvimento de linguagem. Pró-Fono. 2004;16(1):57-66.

18. Rodrigues A, Befi-Lopes DM. Comparação entre as habilidades pragmáticas de crianças normais e crianças com alteração no desenvolvimento de linguagem. Rev Soc Bras Fonoaudiol. 2004;9(2):817.

19. Befi-Lopes DM, Araújo K, Giusti E. Relação entre as habilidades fonológicas e pragmáticas nos distúrbios específicos de linguagem. Pró-Fono. 2005;17(1):45-54.

20. Maillart C, Parisse C. Phonological deficits in French speaking children with SLI. Int J Lang Commun Disord. 2006;41(3):253-74.

21. Wertzner HF. Fonologia. In: Andrade CRF, Befi-Lopes DM, Fernandes FDM, Wertzner HF. ABFW: teste de linguagem infantil nas áreas de fonologia, vocabulário, fluência e pragmática. Barueri: Pró-Fono; 2004b. p. 5-32.

22. Fernandes FDM. Pragmática. In: Andrade CRF, Befi-Lopes DM, Fernandes FDM, Wertzner HF. ABFW: teste de linguagem infantil nas áreas de fonologia, vocabulário, fluência e pragmática. Barueri: PróFono; 2004.

23. Andrade CRF, Befi-Lopes DM, Fernandes FDM, Wertzner HF. ABFW: teste de linguagem infantil nas áreas de fonologia, vocabulário, fluência e pragmática. Barueri: Pró-Fono; 2004.

24. Lamprecht RR. A aquisição da fonologia do português na faixa etária dos 2:9 aos 5:5. Letras Hoje. 1993;28(2):99-106.

25. Yavas MS. Padrões na aquisição da fonologia do português. Letras Hoje. 1998;23(3):7-30.

26. Ingram D. Aspects of phonological acquisition. In: Ingram D. Phonological disability in children. London and Wercester: Guildford; 1976. p. 10-51.

27. Wertzner HF. Articulação: aquisição do sistema fonológico dos três aos sete anos [tese]. São Paulo: Faculdade de Filosofia, Letras e Ciências Humanas da Universidade de São Paulo; 1992.

28. Galea DES. Análise do sistema fonológico em crianças de $2: 1$ a 3:0 anos de idade [dissertação]. São Paulo: Faculdade de Filosofia, Letras e Ciências Humanas da Universidade de São Paulo; 2003.

29. Bishop DV. The underlying nature of specific language impairment. J Child Psychol Psychiatry. 1992;33(1):3-66. Review.

30. Newman RM, McGregor KK. Teachers and laypersons discern quality differences between narratives produced by children with or without SLI. J Speech Lang Hear Res. 2006;49(5):1022-36. 